\title{
Electrical Grounding Improves Vagal Tone in Preterm Infants
}

\author{
Rohit Passi ${ }^{\mathrm{a}}$ Kim K. Doheny ${ }^{\mathrm{a}}$ Yuri Gordin ${ }^{\mathrm{b}}$ Hans Hinssen ${ }^{\mathrm{c}}$ Charles Palmer ${ }^{\mathrm{a}}$ \\ ${ }^{a}$ Division of Newborn Medicine, PennState Health Children's Hospital, and ${ }^{b}$ College of Medicine and ${ }^{c}$ Department \\ of Clinical Engineering, College of Medicine, Pennsylvania State University, Hershey, PA, USA
}

\section{Keywords}

Electromagnetic fields · Vagal tone - Electrical grounding .

Heart rate variability

\begin{abstract}
Background: Low vagal tone (VT) is a marker of vulnerability to stress and the risk of developing necrotizing enterocolitis in preterm infants. Electric fields produced by equipment in the neonatal intensive care unit (NICU) induce an electric potential measurable on the skin in reference to ground. An electrical connection to ground reduces the skin potential and improves VT in adults. Objectives: We aimed to measure the electric field strengths in the NICU environment and to determine if connecting an infant to electrical ground would reduce the skin potential and improve VT. We also wished to determine if the skin potential correlated with VT. Methods: Environmental magnetic flux density (MFD) was measured in and around incubators. Electrical grounding (EG) was achieved with a patch electrode and wire that extended to a ground outlet. We measured the skin potential in 26 infants and heart rate variability in 20 infants before, during, and after grounding. VT was represented by the high-frequency power of heart rate variability. Results: The background MFD in the NICU was below $0.5 \mathrm{mG}$, but it ranged between 1.5 and
\end{abstract}

$12.7 \mathrm{mG}$ in the closed incubator. A 60-Hz oscillating potential was recorded on the skin of all infants. With EG, the skin voltage dropped by about $95 \%$. Pre-grounding VT was inversely correlated with the skin potential. VT increased by $67 \%$ with EG. After grounding, the VT fell to the pre-grounding level. Conclusion: The electrical environment affects autonomic balance. EG improves VT and may improve resilience to stress and lower the risk of neonatal morbidity in preterm infants.

(c) 2017 S. Karger AG, Basel

\section{Introduction}

Equipment in the neonatal intensive care unit (NICU) generates electric fields. The 50 - to $60-\mathrm{Hz}$ alternating current that powers incubators produces extremely-low-frequency electromagnetic fields (ELF-EMFs). The magnetic part of the field within the incubator is between 2 and $100 \mathrm{mG}$ [1-3]. Magnetic fields as low as $2 \mathrm{mG}$ have been shown to produce biological effects on fetuses $[4,5]$ and on cells in culture [6], raising the possibility that these fields may adversely affect the preterm infant.

Bellieni et al. [7] found that vagal tone (VT) in preterm infants reversibly increased when the incubator power 
was switched off [7]. VT is a measure of resilience and homeostasis [8]. Low VT may also be associated with an increased risk of developing necrotizing enterocolitis (NEC) [9].

When a human body is exposed to alternating $60-\mathrm{Hz}$ ELF-EMFs, a voltage is induced on the skin surface. However, when the body is conductively connected to electrical ground, this skin potential is dissipated [10]. Studies in adults found that electrical grounding (EG) produces an increase in VT $[11,12]$.

The primary objective of this study was to determine if the VT of preterm infants in a NICU can be increased by EG. We also wished to measure the magnetic flux density (MFD) within the NICU environment and determine if the skin potential correlated with VT.

\section{Methods}

\section{Subjects and Samples}

This study was conducted in the NICU at the PennState Health Hershey Medical Center from October 2012 to January 2014. The IRB of the Penn State College of Medicine approved the study. The study design was a prospective observational one, using a withinsubjects cross-over approach. We included 26 neonates who were born AGA (appropriately grown for gestational age) and who were 26-36 weeks of postmenstrual age and 6-60 days of age hospitalized in the NICU at the PennState Health Hershey Medical Center. We excluded neonates who were medically unstable, on mechanical ventilation, or born with any severe congenital anomaly or cardiac rhythm disturbance, as these conditions are known to impact autonomic system measurement.

Seventeen patients were initially studied for skin voltage in closed incubators with the power turned on, and 9 in open cribs. Heart rate variability (HRV) data, however, were obtained on 13 infants in closed incubators and on 7 infants in open cribs.

\section{Measurement of ELF-EMFs}

Two empty thermoregulatory units used in the NICU were assessed for MFD only: a closed incubator (Ohmeda Giraffe OmniBed) with the power turned on, and a radiant infant warmer (Ohmeda Ohio Infant Warmer). Additionally, ELF-EMFs emitted from a popular humidifier model, used in conjunction with closed incubator systems, were analyzed. MFD was measured using the Emdex II Gauss Meter (Enertech Consultants, Campbell, CA, USA). The EMF from each incubator was measured in isolation, in a room separate from the NICU environment, without additional AC-powered equipment in proximity. The equipment was plugged into an electrical outlet and left in "standby" mode (power on). The background MFD was recorded prior to each measurement, with a baseline of $0.3-0.5 \mathrm{mG}$ found evenly distributed throughout the plane of the mattress. At 3 -inch increments in both length and width across the mattress surface, the Emdex II meter was used to collect the instantaneous MFD. This instrument was consistently oriented with the display panel in the same direction relative to the incubator while all readings were recorded. A total of 51 points were measured on the oval-shaped surface of the stan- dard incubator mattress; 80 points were measured along the rectangular surface of the mattress in the infant warmer. Three-dimensional representations of the ELF-EMF distributions on the mattresses were generated from these data. We also measured the background MFD in the NICU.

\section{Study Procedure}

The skin potential was measured with a voltmeter (Fluke 725 US Multifunction Process Calibrator) which was connected to the infant's skin and to the dedicated ground socket in the bedside console. We measured the voltage for the first 6 patients with the voltmeter; then, for the next 20 patients, we changed to measuring continuously using a Bioamplifier (Bio Amp Model FE132; ADInstruments, Bella Vista, NSW, Australia) connected to a data acquisition system (Powerlab 16/30 ML 880; ADInstruments) that recorded the signal at $1,000 \mathrm{~Hz}$ so that the oscillating potential could be recorded (LabChart version 7; ADInstruments). We display the results in millivolts. Changes in skin voltage potential were directly recorded upon connection and disconnection from ground in real time. An average of 4 measurements was conducted to calculate the mean skin voltage in the pre-grounding and grounding phases.

\section{Grounding}

To achieve grounding, we used a commercially available patch electrode (Earthing.Com Inc., Thousand Palms, CA, USA) that had a $100-\mathrm{k} \Omega$ resistor built into the snap connector which was connected to the patch on the patient's skin. The resistor provided a safety feature in the unlikely event the infant were exposed to a live electric current. Importantly, the ground outlet was a separate, dedicated outlet on the console at the bedside and had no electrical connection to the power outlets. We reversibly connected the patient to ground by connecting the wire to the ground outlet without handling the baby.

To measure the effect of EG on HRV, the EKG signal from the infant's bedside monitor was digitally captured on the data acquisition system (PowerLab 7) for a duration of 20-40 min while standardizing for the time of day, environmental stimuli, and postfeeding behavioral state. HRV was calculated for each patient by averaging the results of repeated 2-min recordings during epochs which represented the time before, during, and after EG (pregrounding, grounding, and post-grounding phases). EG was discontinued by removal of the wire from the ground outlet without handling the infant. For each epoch of sampling, spectral analysis of HRV was used to determine the HRV parameters.

\section{Heart Rate Variability}

Electrocardiographic R-wave data were obtained by the data acquisition system at a sampling frequency of $1 \mathrm{kHz}$, and stored for later spectral HRV analysis using the PowerLab 7 HRV module. We selected 2-min segments which we manually reviewed to be free of ectopic beats and artifacts for analysis, then averaged the analyses of the epochs for each infant ( $n=4$ segments per infant per phase, i.e., pre-grounding, grounding, and post-grounding). We only measured the low-frequency (LF, indicative of both VT and sympathetic tone) and high-frequency (HF, indicative of VT) components and total power, as the fast Fourier transform of the beat-to-beat intervals used to calculate the frequency measures above is known to have a limited accuracy for power estimation in the very-low-frequency range with segments of duration below 
5 min [13]. The 0.03 - to $0.29-\mathrm{Hz}$ frequency bandwidth was used for measurement of the LF domain and the $0.3-$ to $1.3-\mathrm{Hz}$ bandwidth was used to calculate the HF domain, as it is reflective of the effect of respirations on the heart rate. To calculate the HF bandwidth, we used the mean \pm 2 SD of the spontaneous resting breath rates (20-80 breaths/min) [9]. VT (measured by HF HRV) slows the heart rate during exhalation and prolongs the beat-to-beat interval. The total power spectrum was calculated as the sum of HF $+\mathrm{LF}$, as a measure of overall HRV. To estimate sympathovagal balance, the LF/HF ratio was calculated. The SD of all normal R-R intervals (SDNN) was used as a measure of time domain analysis of HRV as it expresses beat to beat variability. Thus SDNN is an indicator of general HRV and has been used widely in adult studies as marker of cardiovascular and autonomic well-being [14]. HF power is a marker of parasympathetic tone and LF power is a measure of both sympathetic and parasympathetic tone [14].

\section{Statistical Analysis}

Statistical analysis was performed using IBM-SPSS version 24 (SPSS Inc., Armonk, NY, USA). Descriptive statistics were computed for key variables and checked for outliers and normality. Data are expressed as mean \pm SE (or SD) and analyzed with $t$ tests and repeated-measures ANOVAs to compare continuous variables. All tests were two-tailed at a 5\% significance level. Due to skewness of the data on tests of normality, HF and LF, SDNN, and heart rate data were transformed using natural log prior to performing repeated-measures ANOVAs or $t$ tests.

\section{Results}

The subjects included 26 stable preterm infants with a gestational age of $30.35 \pm 2.68$ weeks (mean \pm SD) and a birth weight of $1,476 \pm 488 \mathrm{~g}$ (Table 1). Complete HRV data were available for 20 subjects; on 6 subjects there were only skin voltage data.

The MFD measured within the closed neonatal incubator in our unit (Ohmeda Giraffe OmniBed) ranged from 1.5 to $12.7 \mathrm{mG}$, with a mean of $4.0( \pm 2.8) \mathrm{mG}$. A higher MFD was detected at the end of the mattress, where the power supply and control panel are located (Fig. 1). The MFD at the mattress level from the radiant infant warmer (Ohmeda Ohio Care Plus) was 0.8-1.7 $\mathrm{mG}$, with a mean of $1.1( \pm 0.02) \mathrm{mG}$. Unlike in the closed incubator, there was no appreciable difference in flux across the mattress surface in the radiant infant warmer.

The MFD measured outside and surrounding the closed incubator in standby mode adjacent to the control panel was approximately $280 \mathrm{mG}$, with the highest values near the control panel 2 feet above the floor, at the level of the AC power source. Values above $2 \mathrm{mG}$ were seen up to 2 feet away. The humidifier emitted more than $390 \mathrm{mG}$ adjacent to its rear panel, and values exceeding $2 \mathrm{mG}$ were measured up to 18 inches away. The fields from our trans-

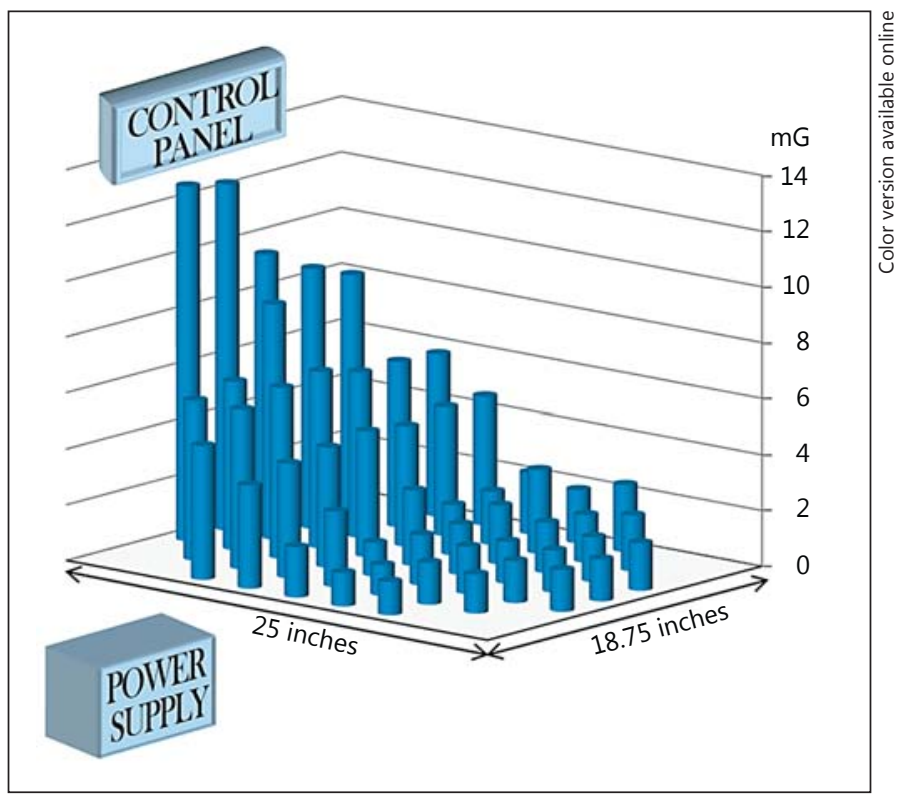

Fig. 1. The columns represent the magnetic flux density (MFD) within the standard incubator measured at the mattress level. The MFD ranged between 1.5 and $12.7 \mathrm{mG}$. The field strength was highest above the power supply and near the control panel.

Table 1. Characteristics of the infant sample $(n=26)$

\begin{tabular}{lrrrr}
\hline Variable & Min. & Max. & Mean & SE \\
\hline GA at birth, weeks & 24.2 & 34.2 & 30.3 & 0.5 \\
DOL on experiment day & 5 & 68 & 22.5 & 3.2 \\
Adjusted GA on experiment day & 31.4 & 35.5 & 33.5 & 0.2 \\
Birth weight, g & 470 & 2,352 & $1,476.2$ & 95.8 \\
Weight on experiment day, g & 955 & 2,241 & $1,721.3$ & 51.3 \\
\hline
\end{tabular}

GA, gestational age; DOL, day of life.

port incubator (Airborne 750i) were nearly 10-fold higher than within the closed incubator: the MFD ranged from 3.6 to $151.8 \mathrm{mG}$. The background levels were $0.3-$ $0.5 \mathrm{mG}$.

We recorded a $60-\mathrm{Hz}$ alternating skin voltage on all babies. The skin voltage dropped the instant the patient was connected to ground (Fig. 2). The skin voltage in the pre-grounding phase was $415.3 \pm 277.5 \mathrm{mV}$ (mean $\pm \mathrm{SD}$ ) and dropped by more than $95 \%$ during the grounding phase to $19.1 \pm 16.8 \mathrm{mV}$ (Table 2). Seventeen infants were placed in a closed incubator with the power on, and 9 infants were placed in an open crib, at the time of the ex- 
Table 2. Changes in skin potential $(\mathrm{mV})$ with electrical grounding

\begin{tabular}{lrlrr}
\hline & Min. & Max. & Mean & \multicolumn{1}{c}{ SE } \\
\hline Pre-grounding phase & 38.92 & $1,414.40$ & 415.29 & 54.4 \\
Grounding phase & 0.94 & 72.05 & 19.06 & 3.3 \\
\hline
\end{tabular}

Twenty-six patients were tested before grounding and again after grounding. Connecting patients to electrical ground produced a $>95 \%$ drop in skin potential.

Table 3. Heart rate variability changes with electrical grounding

\begin{tabular}{|c|c|c|c|}
\hline Variable & $\begin{array}{l}\text { Before } \\
\text { grounding }\end{array}$ & Grounding & $\begin{array}{l}\text { After } \\
\text { grounding }\end{array}$ \\
\hline Total power, $\mathrm{ms}^{2}$ & $81.2(36.59)$ & $126.2(37.48)$ & $69.6(12.18)$ \\
\hline HF power, $\mathrm{ms}^{2}$ & $6.6(1.8)$ & $11.0(3.1)^{*}$ & $6.2(1.2)^{* *}$ \\
\hline LF power, $\mathrm{ms}^{2}$ & $74.6(34.9)$ & $115.3(34.6)^{\S}$ & $63.4(11.5)$ \\
\hline $\mathrm{LF} / \mathrm{HF}$ ratio & $11.3(2.0)$ & $11.69(1.8)$ & $12.5(2.2)$ \\
\hline SDNN, ms & $11.0(1.7)$ & $12.8(1.3)$ & $11.7(1.1)$ \\
\hline Heart rate, bpm & $158.51(2.4)$ & $157.0(2.5)$ & $157.6(2.3)$ \\
\hline
\end{tabular}

Values are expressed as mean (SE); $n=20$. Repeated-measures ANOVA with Bonferroni correction was performed on the natural $\log$ of the raw values shown in the table for HF power, LF power, SDNN, and heart rate. ${ }^{*} p=0.003$, repeated-measures ANOVA before grounding vs. grounding; ${ }^{* *} p=0.02$ grounding vs. after grounding; ${ }^{\circledR} p=0.01$ before grounding vs. grounding. Vagal tone was measured by HF power in $\mathrm{ms}^{2}$. HF, high-frequency; LF, lowfrequency; SDNN, standard deviation of all normal R-R (NN) intervals.

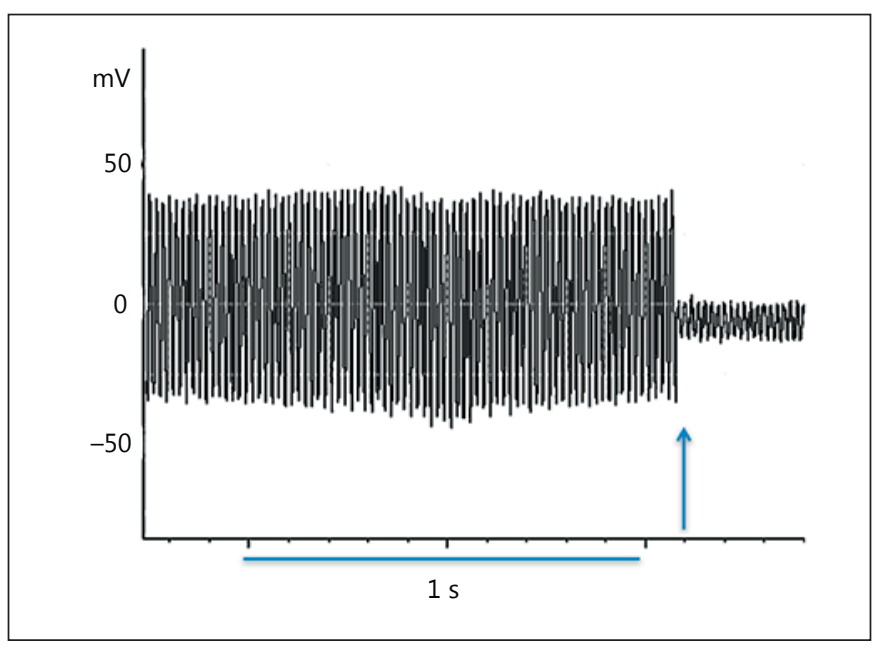

Fig. 2. Recording of skin surface potential. A 1-s interval is shown. The field alternates at $60 \mathrm{~Hz}$. At the onset of electrical grounding (arrow), the voltage diminished immediately. The mean decrease in voltage \pm SE was $95.6 \pm 1.1 \%(p<0.001$, paired sample $t$ test $)$.
Table 4. Comparison of HRV parameters between thermoregulatory units

\begin{tabular}{llll}
\hline HRV parameter & $\begin{array}{l}\text { Open crib } \\
(n=7)\end{array}$ & $\begin{array}{l}\text { Closed incubator } \\
(n=13)\end{array}$ & \\
\hline LF power, ms & & \\
$\quad$ Before grounding & $161(94)$ & $27.9(7)$ & 0.003 \\
$\quad$ Grounding & $192(91)$ & $74.1(17)$ & 0.12 \\
$\quad$ After grounding & $102(24)$ & $42.4(8.1)$ & 0.036 \\
HF power, ms & & & \\
$\quad$ Before grounding & $10.2(4.8)$ & $4.6(0.9)$ & 0.19 \\
$\quad$ Grounding & $16.0(8.6)$ & $8.2(1.4)$ & 0.5 \\
$\quad$ After grounding & $7.8(3.0)$ & $5.3(0.8)$ & 0.56 \\
SDNN, ms & & & \\
$\quad$ Before grounding & $17.3(3.4)$ & $7.6(1)$ & 0.001 \\
$\quad$ Grounding & $15.7(2.8)$ & $11.2(1.1)$ & 0.12 \\
$\quad$ After grounding & $15.9(1.9)$ & $9.5(0.9)$ & 0.008 \\
Heart rate, bpm & & & \\
$\quad$ Before grounding & $155(3.7)$ & $160(3.1)$ & 0.65 \\
$\quad$ Grounding & $154(4.4)$ & $158(3.2)$ & 0.82 \\
$\quad$ After grounding & $155(4.3)$ & $159(2.8)$ & 0.81 \\
\hline
\end{tabular}

Values are expressed as mean (SE). Comparison of HRV parameters between 7 infants in open cribs compared with 13 infants in closed incubators with heat on. Independent $t$ tests were done on the natural log-transformed data of all the parameters displayed. $p<0.05$ is significant. LF, low-frequency; HF, high-frequency; HRV, heart rate variability; SDNN, standard deviation of beat-to-beat intervals between normal heart beats.

periment. The skin voltage on the 17 infants in the closed incubators during the pre-grounding phase was $483 \pm 306$ $\mathrm{mV}$, compared to $288 \pm 159 \mathrm{mV}$ (mean \pm SD) on the 9 infants in the open crib $(p=0.09)$. Interestingly, there was a significant inverse correlation between the skin potential and HF power in the pre-grounding phase $(R=0.47$, $p=0.04)$. Accordingly, higher skin voltage was associated with lower VT.

The HF power for the 20 subjects increased by $67 \%$ when they were connected to ground (Table 3). The HF power was $6.59 \mathrm{~ms}^{2}$ in the pre-grounding phase; it increased to $11.00 \mathrm{~ms}^{2}$ upon grounding $(p=0.003)$ and decreased to $6.14 \mathrm{~ms}^{2}$ upon discontinuation of grounding $(p=0.02)$ (repeated-measures ANOVA).

Grounding produced a significant increase in LF power $(p=0.01)$, which fell again in the post-grounding phase. The LF/HF ratio did not change significantly during grounding. The SDNN and heart rate also did not change significantly with grounding (Table 3 ).

We compared the effect of the thermoregulatory environment on the HRV parameters before, during, and af- 
ter grounding. Interestingly, we found that the infants in closed incubators had lower LF power and SDNN values than the infants in open cribs before and after grounding. During grounding there were no between-group differences in any of the HRV parameters (Table 4).

\section{Discussion}

This study showed that EG of preterm infants produced a significant increase in VT. VT reverted to the pre-grounding level when EG was discontinued. The increase in LF power may also reflect an increase in parasympathetic tone, as LF power combines sympathetic and parasympathetic components. This study confirms an earlier report which found that EG produces an increase in VT in adults [12]. This is the first time that EG has been studied in the newborn.

Our study also confirmed a $60-\mathrm{Hz}$ skin potential on our preterm patients, as described by Applewhite [10]. This oscillating potential is induced by the ELF-EMF in the electrical environment. We found that the infants' VT before being connected to ground was inversely proportional to the skin potential. We also found that the skin potential dropped immediately as the patients were connected to ground. We found lower skin potentials in those infants that were cared for in open cribs compared to closed incubators; however, the study was not powered to reveal any significance. There was a significantly lower LF power and lower SDNN in the infants in closed incubators compared to those in open cribs before and after grounding. This effect was not seen with grounding, suggesting that the between-group differences may have been related to the influence of environmental electric fields, which were higher in the closed incubators. We did not test any infants that were in radiant warmers.

VT in preterm infants is a valuable marker of vulnerability to stress [8]. Our own experience showed that the risk of developing NEC increases by a factor of 10 for every SD decrease in HF power [9]. A causative link between low VT and NEC may be plausible, as VT has an anti-inflammatory effect. Specifically, efferent vagal activity on macrophages in the spleen and gastrointestinal tract downregulates the production of inflammatory cytokines in response to inflammation [15]. Systemic inflammation is linked to neurodevelopmental delay in the preterm infant [16]; therefore, strategies that dampen inflammation are clinically relevant.

The background MFD levels in the NICU were less than $0.5 \mathrm{mG}$ and were similar to general household levels.
The levels found in our incubators were lower than those reported more than 10 years ago and may reflect an improved engineering approach to incubator design $[3,17]$. We confirmed that the MFD was very much dependent on proximity to the power source. If we position infants away from the power sources within incubators, we could reduce their exposure to ELF-EMF 8-fold.

Epidemiological studies performed during pregnancy showed increasing risks of asthma and obesity for the developing fetus when the expectant mother is exposed to a daily MFD of $2 \mathrm{mG}[4,5]$, and the risk of developing childhood leukemia doubles with postnatal exposure above $4 \mathrm{mG}$ [18]. Accordingly, even though more than $2 \mathrm{mG}(>0.2 \mu \mathrm{T})$ is associated with negative health consequences on the growing fetus [4-6], values exceeding this value are still commonly seen within our incubators and also within 18 inches from equipment such as incubator humidifier control panels.

An animal study showed that harmful effects of LFEMFs are mediated by oxidative injury to brain cells; antioxidants can block these effects [19]. EG makes an electrical connection to the surface of the earth, which is known to possess a supply of free, mobile electrons. These electrons are continuously replenished by the global atmospheric electrical circuit, where the sources of electrons are thunderstorms and lightning strikes [20,21]. When the body is grounded, the body's electric skin potential becomes equalized with the earth's electric potential through a transfer of electrons from the earth to the body [20]. This virtually eliminates the $60-\mathrm{Hz}$ potential on the surface of the body induced by the electrical environment, and thus may prevent the fields from producing electrical perturbations of molecules inside the body. Secondly, it is hypothesized that grounding provides access to an abundant, immediate source of mobile electrons as natural antioxidants $[22,23]$.

\section{Disclosure Statement}

K.K.D. and C.P. receive salary support for research by the National Institute of Diabetes and Digestive and Kidney Diseases (NIDDK) of the National Institutes of Health under award No. 1R01DK099350 (K.K.D.). C.P. receives salary support from the University City Science Center. None of the funding sources had any role in the design of the study, in the analysis and interpretation of the data, in the decision to submit the manuscript, or in the preparation, review, or approval of the manuscript. 


\section{References}

1 Bearer CF: Electromagnetic fields and infant incubators. Arch Environ Health 1994;49: 352-354.

2 Bullough J, Rea MS, Stevens RG: Light and magnetic fields in a neonatal intensive care unit. Bioelectromagnetics 1996;17:396-405.

3 Cermáková E: Study of extremely low frequency electromagnetic fields in infant incubators. Int J Occup Med Environ Health 2003; 16:215-220.

4 Li DK, Chen H, Odouli R: Maternal exposure to magnetic fields during pregnancy in relation to the risk of asthma in offspring. Arch Pediatr Adolesc Med 2011;165:945-950.

5 Li DK, Ferber JR, Odouli R, Quesenberry CP Jr: A prospective study of in-utero exposure to magnetic fields and the risk of childhood obesity. Sci Rep 2012;2:540.

6 Johansson O: Disturbance of the immune system by electromagnetic fields - a potentially underlying cause for cellular damage and tissue repair reduction which could lead to disease and impairment. Pathophysiology 2009 16:157-177.

7 Bellieni CV, Acampa M, Maffei M, Maffei S, Perrone S, Pinto I, Stacchini N, Buonocore G: Electromagnetic fields produced by incubators influence heart rate variability in newborns. Arch Dis Child Fetal Neonatal Ed 2008 93:F298-F301.

8 Porges SW: Vagal tone: a physiologic marker of stress vulnerability. Pediatrics 1992;90(pt 2): 498-504.
9 Doheny KK, Palmer C, Browning KN, Jairath P, Liao D, He F, Travagli RA: Diminished vagal tone is a predictive biomarker of necrotizing enterocolitis-risk in preterm infants. Neurogastroenterol Motil 2014;26:832-840.

10 Applewhite R: The effectiveness of a conductive patch and a conductive bed pad in reducing induced human body voltage via the application of earth ground. Eur Biol Bioelectromagnetics 2005;1:23-40.

11 Ghaly M, Teplitz D: The biologic effects of grounding the human body during sleep as measured by cortisol levels and subjective reporting of sleep, pain, and stress. J Altern Complement Med 2004;10:767-776.

12 Chevalier G, Sinatra ST: Emotional stress, heart rate variability, grounding and improved autonomic tone: clinical applications. Integr Med 2011;10:16-21.

13 Rosen H, Craelius W, Curcie D, Hiatt M, Hegyi T: Spectral analysis of heart variability in the newborn infant. Biol Neonate 2000;77: 224-229.

14 American Heart Association (AHA) Task Force of the European Society of Cardiology the North American Society of Pacing Electrophysiology: Heart rate variability: standards of measurement, physiological interpretation, and clinical use. Circulation 1996; 93:1043-1065.

15 Tracey KJ: Physiology and immunology of the cholinergic antiinflammatory pathway. J Clin Invest 2007;117:289-296.

16 Strunk T, Inder T, Wang X, Burgner D, Mallard C, Levy O: Infection-induced inflammation and cerebral injury in preterm infants. Lancet Infect Dis 2014;14:751-762.
17 Riminesi C, Andreuccetti D, Fossi R, Pezzati $\mathrm{M}$ : ELF magnetic field exposure in a neonatal intensive care unit. Bioelectromagnetics 2004; 25:481-491.

18 Ahlbom IC, Cardis E, Green A, Linet M, Savitz D, Swerdlow A; ICNIRP (International Commission for Non-Ionizing Radiation Protection) Standing Committee on Epidemiology: Review of the epidemiologic literature on EMF and health. Environ Health Perspect 2001;109(suppl 6):911-933.

19 Lai H, Singh NP: Magnetic-field-induced DNA strand breaks in brain cells of the rat. Environ Health Perspect 2004;112:687-694.

20 Chevalier G, Sinatra ST, Oschman JL, Sokal K, Sokal P: Earthing: health implications of reconnecting the human body to the Earth's surface electrons. J Environ Public Health 2012;2012:291541.

21 Anisimov SV, Mareev EA, Bakastov SS: On the generation and evolution of aeroelectric structures in the surface layer. J Geophys Res Atmos 1999;104:14359-14367.

22 Oschman JL: Can electrons act as antioxidants? A review and commentary. J Altern Complement Med 2007;13:955-967.

23 Oschman JL, Chevalier G, Brown R: The effects of grounding (earthing) on inflammation, the immune response, wound healing, and prevention and treatment of chronic inflammatory and autoimmune diseases. J Inflamm Res 2015;8:83-96. 